\title{
openheart Predictors of medium-term mortality in patients hospitalised with coronary artery disease in a resource-limited South-East Asian setting
}

\author{
Andriany Qanitha, ${ }^{1,2}$ Cuno S. P. M. Uiterwaal, ${ }^{3}$ Jose P. S. Henriques, ${ }^{4}$ \\ Idar Mappangara, ${ }^{5}$ Irfan Idris, ${ }^{2}$ Muzakkir Amir, ${ }^{5}$ Bastianus A. J. M. de Mol ${ }^{1}$
}

To cite: Qanitha A,

Uiterwaal CSPM.,

Henriques JPS., et al. Predictors of medium-term mortality in patients hospitalised with coronary artery disease in a resource-limited South-East Asian setting. Open Heart 2018;5:e000801. doi:10.1136/ openhrt-2018-000801

The abstract of this paper was presented at the 21st Asian Pacific Society of Cardiology (APSC) Congress, 13-15 July 2017, in Singapore.

Received 14 February 2018 Revised 24 May 2018 Accepted 13 June 2018

Check for updates

(c) Author(s) (or their employer(s)) 2018. Re-use permitted under CC BY-NC. No commercial re-use. See rights and permissions. Published by BMJ.

For numbered affiliations see end of article.

Correspondence to

Andriany Qanitha; a.qanitha@ amc.uva.nl; myaqanitha@gmail. com

\section{ABSTRACT}

Objective To measure medium-term outcomes and determine the predictors of mortality in patients with coronary artery disease (CAD) both during and after hospitalisation in a resource-limited South-East Asian setting.

Methods From February 2013 to December 2014, we conducted a prospective observational cohort study of 477 patients admitted to Makassar Cardiac Center, Indonesia, with acute coronary syndrome and stable CAD. We actively obtained data on clinical outcomes and afterdischarge management until April 2017. Multivariable Cox proportional hazard analysis was performed to examine predictors for our primary outcome, all-cause mortality.

Results From hospital admission, patients were followed over a median of 18 (IQR 6-36) months; in total 154 (32.3\%) patients died. More patients with acute myocardial infarction died in the hospital compared with patients with unstable and stable angina $(p=0.002)$. Over the total follow-up, there was a difference in mortality between non-ST-segment elevation myocardial infarction $(n=41$, 48.2\%), ST-segment elevation myocardial infarction $(\mathrm{n}=65,30.8 \%)$, unstable angina $(\mathrm{n}=18,26.5 \%)$ and stable coronary artery disease $(n=30,26.5 \%)$ groups $(p=0.007)$. The independent predictors of all-cause mortality were hyperglycaemia on admission (HR 1.55 (95\% Cl 1.12 to 2.14), $p=0.008$ ), heart failure/Killip class $\geq 2$ (HR 2.50 (95\% Cl 1.76 to 3.56), $\mathrm{p}<0.001$ ), estimated glomerular filtration rate $<60 \mathrm{~mL} / \mathrm{min}$ (HR 1.77 (95\% Cl 1.26 to 2.50 ), $\mathrm{p}=0.001$ ), no revascularisation (percutaneous coronary intervention/coronary artery bypass grafting) (HR 2.38 (95\% $\mathrm{Cl} 1.31$ to 4.33), $\mathrm{p}=0.005$ ) and poor adherence to after-discharge medications (HR 10.28 (95\% Cl 5.52 to 19.16), $\mathrm{p}<0.001)$. Poor medication adherence predicted postdischarge mortality and did so irrespective of underlying $\mathrm{CAD}$ diagnosis ( $\mathrm{p}$ interaction $=0.88$ ).

Conclusions Patients with CAD in a poor South-East Asian setting experience high in-hospital and mediumterm mortality. The initial severity of the disease, lack of access to guidelines-recommended therapy and poor adherence to after-discharge medications are the main drivers for excess mortality. Improved access to early and late hospital care and patient education should be prioritised for better survival.

\section{Key questions}

What is already known about this subject?

- Registry data on cardiovascular diseases and deaths in the low-income and middle-income countries are lacking.

- Previous studies have suggested that traditional risk factors and adverse clinical presentation on admission were all independent predictors of cardiovascular death.

- Evidence showed that well-established traditiona risk factors in Western and high-income countries play a similar role in cardiovascular mortality in the South-East Asian region.

What does this study add?

- Mortality both during and after hospitalisation is unacceptably high in patients with coronary artery disease (CAD) in Indonesia; after the total follow-up (median 18 (IQR 6-36) months), $32.3 \%$ of the patients died.

- In-hospital and total medium-term mortality differed between the underlying diseases (higher in non-STsegment elevation myocardial infarction (NSTEMI) than otherdiagnosis groups), with $p=0.002$ and $p=0.007$, respectively.

- The initial severity of the disease, lack of access to guidelines-recommended therapy and poor adherence to after-discharge medications are the main drivers for excess medium-term mortality.

- Poor adherence to medications predicted after-discharge mortality and did so irrespective of underlying $\mathrm{CAD}$ diagnosis ( $\mathrm{p}$ interaction $=0.88$ ).

How might this impact on clinical practice?

- This study provides a general picture of risk stratification for clinical practitioners to recognise patients with CAD with poor prognosis in a resource-limited South-East Asian setting, particularly in Indonesia.

- Hospitalisation is an important window of opportunity to address both in-hospital and, largely as a consequence, longer term survival in patients with CAD.

- Optimising medication adherence and lifestyle adjustment, irrespective of the underlying CAD diagnosis, will likely help reduce excess CAD mortality. 


\section{INTRODUCTION}

The rate of cardiovascular disease (CVD) and death is substantially higher in low-income and middle-income countries (LMICs) than in high-income countries. ${ }^{1}$ It is estimated that globally, more than $80 \%$ of cardiovascular deaths occur in LMICs. ${ }^{1}$ Half of the world's CVD burden is expected to occur in Asia. ${ }^{2}$ Also, the prevalence of heart failure is higher in South-East Asian countries than in the rest of the world. ${ }^{3}$ The WHO reported that in South-East Asian populations, the prevalence of CVD risk factors in young adults is high as is the rate of premature death (age $<60$ years). ${ }^{4}$ Despite the alarming increase of cardiovascular deaths in these regions, identification of risk factors associated with medium-term mortality in patients with coronary artery disease (CAD) in the resource-limited South-East Asian countries is poorly documented. This is particularly true for Indonesia with its 260 million inhabitants, which has a poor healthcare infrastructure, resulting in a lack of registration of CVD and death.

Epidemiological studies have shown that risk factors for recurrent non-fatal and fatal CAD resemble risk factors for primary events. ${ }^{5}$ Several studies have suggested that the unmodifiable or uncontrollable traditional CVD risk factors (eg, older age, ${ }^{6}$ diabetes mellitus ${ }^{56}$ or hyperglycaemia, ${ }^{7}$ smoking, ${ }^{5}$ and systolic blood pressure ${ }^{8}$ ), as well as adverse clinical profiles on hospital admission, ${ }^{68}$ were all independent predictors of CVD deaths. Yet these previous studies were conducted in Western populations with supposedly optimal follow-up treatment and disease management. There is substantial evidence to assume that traditional risk factors that are well-established in high-income countries play a similar role in CVD mortality in the South-East Asian region. However, both different levels of exposure to such risk factors and other additional factors may explain local excess mortality. Therefore, we questioned if predictors of mortality, particularly modifiable factors, in patients admitted to the hospital for CAD are different in the SouthEast Asian setting. This is the first study to fully report on medium-term mortality after hospital discharge in Indonesia. We conducted a prospective cohort study of patients with $\mathrm{CAD}$ in Makassar to evaluate medium-term clinical outcomes and to investigate what factors particularly impact on all-cause mortality in patients with $\mathrm{CAD}$ during and after hospitalisation, in a country with limited infrastructural, financial and human resources.

\section{METHODS}

\section{Study population}

In this prospective observational cohort study, we included 477 consecutive patients with CAD who presented between February 2013 and December 2014 at Makassar Cardiac Center, Wahidin Sudirohusodo Hospital, one of the two public cardiac referral centres in East Indonesia. This cardiac centre mainly serves the 9.5 million South Sulawesi population and also other regions inside and outside Sulawesi island. The catchment area of Makassar Cardiac Center is shown in figure 1.

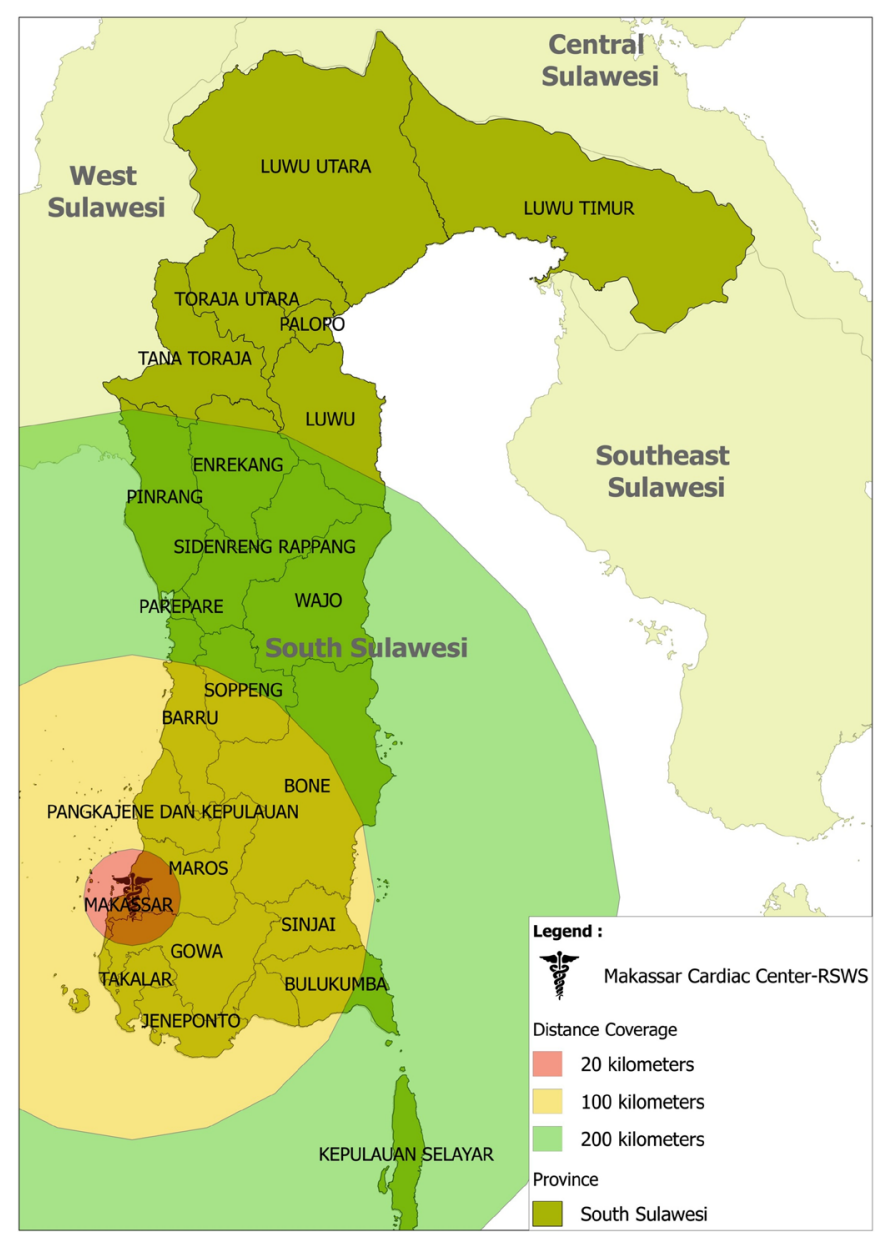

Figure 1 Province of South Sulawesi (46,717 km2) with its cities and regencies - catchment area of Makassar Cardiac Center, Rumah Sakit Wahidin Sudirohusodo.

Patients were included if they had been clinically diagnosed with $\mathrm{CAD}$, defined as acute coronary syndrome (ACS) or stable coronary artery disease (SCAD). ACS was defined as unstable angina (UA), non-ST-segment elevation myocardial infarction (NSTEMI) or ST-segment elevation myocardial infarction (STEMI).$^{9} 10$ SCAD or stable angina included prior ACS, prior coronary revascularisation or known significant CAD on angiography without revascularisation. ${ }^{11}$ To enrol as a participant, all eligible patients signed written informed consent. We excluded all fatal patients who immediately died at the emergency department or intensive cardiovascular care unit (ICCU) before giving informed consent and all patients with normal coronary angiography (CAG) (defined as $0 \%$ lumen stenosis in all coronary vessels). ${ }^{12}$ The flow chart of the study population is presented in figure 2.

\section{Data collection and follow-up}

On hospital admission, we obtained baseline clinical data from medical records and questionnaire interviews. Data on sociodemographic characteristics, lifestyle, dietary pattern, family history of CVD and premature sudden 


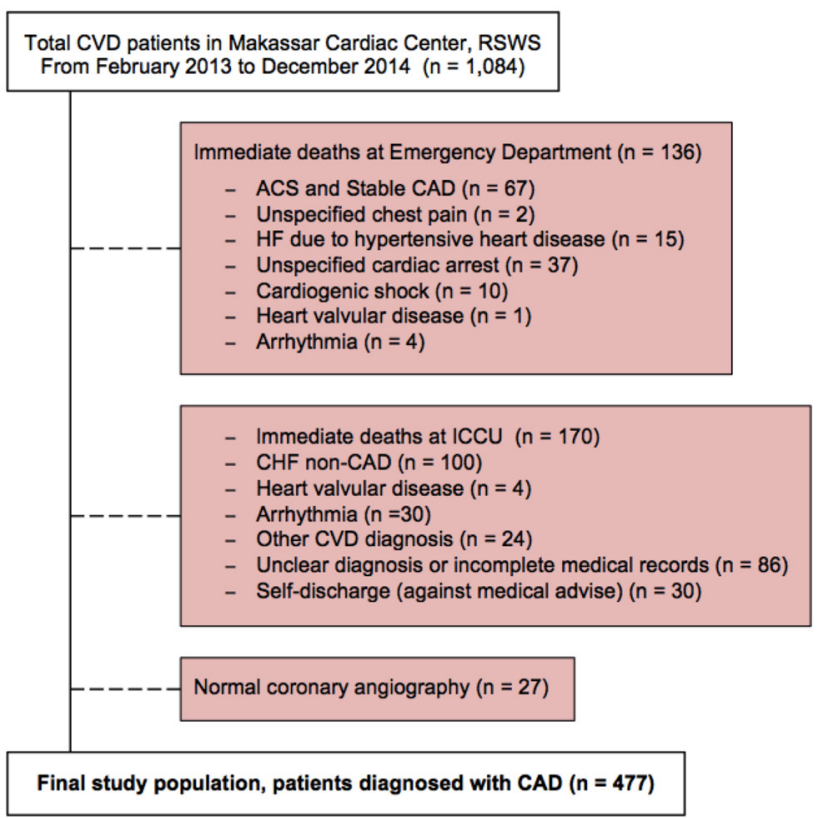

Figure 2 Overview of the study population. ACS, acute coronary syndrome; CAD, coronary artery disease; $\mathrm{CHF}$, congestive heart failure; CVD, cardiovascular disease; ICCU, intensive cardiovascular care unit; HF, heart failure; RSWS, Rumah Sakit Wahidin Sudirohusodo (Wahidin Sudirohusodo Hospital).

death at age $<60$ years ${ }^{13}$ and clinical profiles were all obtained.

Prospectively collected clinical data on physical examination covered electrocardiography, echocardiography, CAG and laboratory tests including cardiac enzymes. In-hospital management and at-discharge medications were also recorded. Plasma glucose, lipid profiles, uric acid, and renal and liver functions were measured within 24hours of hospital admission following a minimum 8-hour fast in all participants. All blood samples were analysed in the hospital laboratory using standardised methods. Hyperglycaemia on admission was defined as fasting plasma glucose $>7.7 \mathrm{mmol} / \mathrm{L}$ on hospital admission. $^{7}$

The endpoint in this study was all-cause mortality. We actively collected these data during hospitalisation, at 30 days, 6 months and 12 months, and yearly up to 3 years or the end of follow-up period on 30 April 2017. During home visits or phone call interviews, research nurses recorded the dates and causes of participants' deaths. However, the cause of death was not specified, largely due to the lack of proper death records in Indonesia, with most of the deaths occurring suddenly at home.

We obtained self-reported data on postdischarge management, including adherence to medications (yes/ no), names or types of medications, as well as lifestyle modifications. This included smoking cessation (yes/ no) and dietary habit improvement, that is, restriction from salt/monosodium glutamate-containing food and fatty/deep-fried food (yes/no). At 30-day follow-up, we collected data on medications from all survivors $(n=406)$ and smoking status of active smokers at baseline $(n=108)$. Meanwhile, data on dietary changes were collected at 3 years or at the end of study follow-up $(n=316)$.

\section{Statistical analysis}

Categorical variables were expressed as numbers (percentage), and continuous variables as mean \pm SD. Skewed data were presented as median (Q1-Q3). Baseline characteristics, clinical presentations and management in hospital and after discharge were tabulated by ACS (STEMI, NSTEMI and UA) and non-ACS (SCAD).

We constructed a Kaplan-Meier curve to depict the cumulative incidence of all-cause mortality among patients with CAD. Follow-up time was defined as the time from date of hospital admission to death or censoring, until the end of study follow-up. The survival times of participants who had moved, who had given an incorrect address, and who, for other reasons, were unreachable via telephone or home visit were censored at the last date that they were known to be still alive. Survival times of participants who were still alive at the end of follow-up were censored on 30 April 2017.

Multivariable Cox regression analysis was performed to estimate HRs for all selected predictors of medium-term, all-cause mortality among patients with CAD. A $95 \%$ CI not including 1, corresponding to a two-sided $\mathrm{p}$ value $<0.05$, was considered statistically significant. We performed all statistical analyses with SPSS V.23.0.

\section{RESULTS}

Among 477 patients hospitalised with CAD, a total of 347 $(72.7 \%)$ were male and the mean age was $58.2 \pm 10.8$ years.

Table 1 shows the baseline characteristics of patients with ACS (STEMI, NSTEMI and UA) and SCAD. Patients with ACS were relatively younger, had higher plasma glucose, lower income and educational attainment levels, and were more often current smokers compared with patients with SCAD. More than half (51.2\%) of our patients were coming from outside Makassar city: 121 (25.4\%) patients lived between 20 and $100 \mathrm{~km}$ from the hospital; 48 (10.1\%) between 100 and $200 \mathrm{~km}$; and 75 $(15.7 \%)$ more than $200 \mathrm{~km}$.

The clinical profiles and in-hospital treatments per diagnosis are presented in table 2. A total of 358 patients $(75.1 \%)$ had a history of hypertension, particularly the NSTEMI and UA groups. The STEMI group had the shortest time interval between angina onset and hospital admission (24 (IQR 7-48) hours), compared with the other groups. Duration of hospitalisation and time from admission to intervention were significantly shorter in the SCAD group than in the ACS group. Of 211 patients with STEMI, only $10(4.7 \%)$ underwent primary percutaneous coronary intervention (PCI) and $13(6.2 \%)$ received thrombolysis for early reperfusion, and $30(14.2 \%)$ underwent elective PCI or coronary artery bypass grafting (CABG). 
Table 1 Baseline characteristics of all patients according to diagnosis

\begin{tabular}{|c|c|c|c|c|c|c|}
\hline & STEMI & NSTEMI & UA & SCAD & Total & \\
\hline Variables & $(n=211)$ & $(n=85)$ & $(n=68)$ & $(n=113)$ & $(n=477)$ & P values \\
\hline Male sex & $161(76.3)$ & $51(60.0)$ & 48 (70.6) & $87(77.0)$ & 347 (72.7) & 0.023 \\
\hline Age (years) & $56.6 \pm 11.8$ & $58.6 \pm 10.5$ & $58.7 \pm 10.2$ & $60.6 \pm 8.8$ & $58.2 \pm 10.8$ & 0.016 \\
\hline Previous hypertension & $142(67.3)$ & $71(83.5)$ & $55(80.9)$ & $90(79.6)$ & $358(75.1)$ & 0.006 \\
\hline Previous MI & $61(28.9)$ & 35 (41.2) & $30(44.1)$ & $38(33.6)$ & $164(34.4)$ & 0.06 \\
\hline Diabetes mellitus & $62(29.4)$ & $35(41.2)$ & $15(22.1)$ & $35(31.0)$ & $147(30.8)$ & 0.074 \\
\hline Waist circumference (cm) & $84.9 \pm 6.1$ & $83.7 \pm 10.9$ & $84.8 \pm 5.6$ & $84.9 \pm 6.1$ & $84.6 \pm 7.1$ & 0.575 \\
\hline Obese $\left(\mathrm{BMl} \geq 25 \mathrm{~kg} / \mathrm{m}^{2}\right)$ & $66(31.3)$ & $32(37.6)$ & $21(30.9)$ & $46(40.7)$ & $165(34.6)$ & 0.301 \\
\hline Metabolic syndrome* & $118(55.9)$ & $56(65.9)$ & $34(50.0)$ & $56(49.6)$ & $264(55.3)$ & 0.104 \\
\hline Current smoker & $72(34.1)$ & 18 (21.2) & $13(19.1)$ & $20(17.7)$ & $123(25.8)$ & 0.003 \\
\hline Former smoker & $62(29.4)$ & 27 (31.8) & $29(42.6)$ & $56(49.6)$ & $174(36.5)$ & 0.002 \\
\hline Parental history of CVD & 42 (19.9) & $26(30.6)$ & $27(39.7)$ & $27(23.9)$ & $122(25.6)$ & 0.007 \\
\hline Physical inactivity & $129(61.1)$ & $46(54.1)$ & $43(63.2)$ & $59(52.2)$ & $277(58.1)$ & 0.296 \\
\hline$>20 \mathrm{~km}$ from the hospital & $124(58.8)$ & $40(47.1)$ & $29(42.6)$ & $54(47.8)$ & $247(51.8)$ & 0.049 \\
\hline Low monthly income $†$ & $116(55.0)$ & $60(70.6)$ & $33(48.5)$ & $39(34.5)$ & $248(52.0)$ & $<0.001$ \\
\hline Low education $\ddagger$ & $71(33.6)$ & $31(36.5)$ & $18(26.5)$ & $18(15.9)$ & $138(28.9)$ & 0.003 \\
\hline
\end{tabular}

Values are $\mathrm{n}(\%)$ or mean \pm SD. Comparison was done using one-way analysis of variance test for continuous variables and Pearson's $\chi^{2}$ test for categorical variables.

*Defined using the National Cholesterol Education Programme - Adult Treatment Panel III classification.

†Defined as monthly income $<1,810,000$,- Indonesian Rupiah (US $\$ 1=13500$ IDR). The cut point was based on the national average of the minimum income for proper life in 2015.

‡Defined as the highest formal education: junior high/elementary school, never school or illiterate.

BMI, body mass index; CVD, cardiovascular disease; MI, myocardial infarction; NSTEMI, non-ST-elevation myocardial infarction; SCAD,

stable coronary artery disease; STEMI, ST-elevation myocardial infarction; UA, unstable angina.

Adherence to medications and lifestyle adjustments after hospital discharge in all survivors is cross-tabulated in table 3. Of 406 survivors in the first 30 days, only $44.1 \%$ then adhered to after-discharge medications. At 30-day follow-up, more than half of the survivors had improved their lifestyles by quitting smoking, and implementing low-cholesterol and low-sodium diet.

In table 4, we estimated the fully adjusted Cox regression models for medication adherence as predictor of mortality, both within the subgroups of the types of diagnosed CAD and for separate sections of follow-up. Adherence was significantly associated with mortality in all CAD subgroups, and both for total follow-up and for follow-up after hospital discharge. Formal testing with an interaction term medication adherence $\times$ type of CAD diagnosis in a fully adjusted model with all patients showed no statistically significant interaction $(\mathrm{p}=0.88)$.

Over the whole follow-up period, a total of 154 (32.3\%) participants had died. The mean age of the deceased patients was 60.8 years. Cumulative mortality differed between the categories: NSTEMI (48.2\%), STEMI $(30.8 \%)$, UA $(26.5 \%)$ and SCAD $(26.5 \%)$ (see figure 3$)$. Figure 4 shows Kaplan-Meier curves for short-term and medium-term mortality with considerably higher rates in patients with NSTEMI compared with other CAD diagnoses $(\mathrm{p}=0.001$ and $\mathrm{p}=0.037$, respectively).
The forest plot of multivariable HRs for medium-term mortality among patients with CAD is shown in figure 5 . The independent predictors of medium-term, all-cause mortality of patients with CAD were hyperglycaemia on admission (HR 1.55 (95\% CI 1.12 to 2.14), $\mathrm{p}=0.008$ ), concomitant heart failure or Killip class $\geq 2$ (HR 2.50 (95\% CI 1.76 to 3.56), $\mathrm{p}<0.001$ ), reduced renal function (estimated glomerular filtration rate (eGFR) $<60 \mathrm{~mL} /$ min; HR 1.77 (95\% CI 1.26 to 2.50), $\mathrm{p}=0.001$ ), absence of revascularisation (PCI or CABG) (HR 2.38 (95\% CI 1.31 to 4.33$), \mathrm{p}=0.005)$ and poor adherence to medications (HR 10.28 (95\% CI 5.52 to 19.16), $\mathrm{p}<0.001$ ).

Seven $(1.5 \%)$ participants were lost to follow-up. The median of total follow-up was 18 (IQR 6-36) months.

\section{DISCUSSION}

The main finding of our unique cohort is that mortality both during hospitalisation and after hospital discharge in Indonesia is high in patients with CAD. During the whole follow-up period (median 18 (IQR 6-36) months), $154(32.3 \%)$ patients died. Many patients presented with poor clinical conditions (ie, hyperglycaemia, concomitant heart failure/Killip class $\geq 2$ and reduced renal function) and had difficult access to guidelines-recommended therapy (ie, untreated with early thrombolysis or primary PCI for ACS) during 
Table 2 Clinical profiles and treatments of all patients according to diagnosis

\begin{tabular}{|c|c|c|c|c|c|}
\hline & STEMI & NSTEMI & UA & SCAD & \\
\hline Variables & $(n=211)$ & $(n=85)$ & $(n=68)$ & $(n=113)$ & $P$ values \\
\hline Systolic BP (mm Hg) & $127.7 \pm 25.1$ & $131 \pm 33.1$ & $136.1 \pm 27.0$ & $127.3 \pm 20.4$ & 0.089 \\
\hline Diastolic BP (mm Hg) & $82.0 \pm 15.3$ & $82.0 \pm 18.6$ & $85.9 \pm 14.7$ & $80.8 \pm 10.3$ & 0.152 \\
\hline Plasma glucose $(\mathrm{mmol} / \mathrm{L})^{*}$ & $7.4(6.2-9.9)$ & $7.8(6.0-9.8)$ & $6.2(5.3-8.4)$ & $5.9(5.1-8.2)$ & $<0.001$ \\
\hline Total cholesterol (mmol/L) & $5.0(4.2-5.8)$ & $4.9(4.2-5.5)$ & $4.9(4.2-5.6)$ & $5.1(3.7-5.6)$ & 0.633 \\
\hline Triglycerides (mmol/L)* & $1.4(1.0-1.8)$ & $1.6(1.1-1.9)$ & $1.4(1.0-2.2)$ & $1.5(1.0-1.9)$ & 0.864 \\
\hline HDL-chol (mmol/L) & $0.95 \pm 0.29$ & $0.91 \pm 0.35$ & $0.97 \pm 0.31$ & $0.92 \pm 0.35$ & 0.562 \\
\hline LDL-chol (mmol/L) $)^{*}$ & $3.4(2.6-4.0)$ & $3.2(2.6-4.1)$ & $3.1(2.6-3.9)$ & $3.2(2.3-3.7)$ & 0.157 \\
\hline $\mathrm{LVEF} \leq 35 \%$ & $30(23.8)$ & $12(26.1)$ & $7(18.4)$ & $14(26.9)$ & 0.801 \\
\hline Atrial fibrillation & $2(0.9)$ & $4(4.7)$ & $3(4.4)$ & $3(2.7)$ & 0.188 \\
\hline LVH & $65(30.8)$ & $35(41.2)$ & $24(35.3)$ & $21(18.6)$ & 0.005 \\
\hline Troponin T $(\mu \mathrm{g} / \mathrm{L})^{*}$ & $0.86(0.20-2.00)$ & $0.35(0.15-0.78)$ & $0.02(0.02-0.24)$ & $0.00(0.00-0.02)$ & $<0.001$ \\
\hline eGFR $<60 \mathrm{~mL} / \mathrm{min}$ & $59(28.0)$ & $36(42.4)$ & $13(19.1)$ & $41(36.3)$ & 0.008 \\
\hline With HF or Killip class $\geq 2$ & $80(37.9)$ & $48(56.5)$ & $22(32.4)$ & $34(30.1)$ & 0.001 \\
\hline With concomitant stroke $\dagger$ & $9(4.3)$ & $9(10.6)$ & $0(0.0)$ & $3(2.7)$ & 0.008 \\
\hline With cardiogenic shock $†$ & $14(6.6)$ & $3(3.5)$ & $0(0.0)$ & $0(0.0)$ & 0.005 \\
\hline Multivessel disease $\neq$ & $63(29.9)$ & $18(21.2)$ & $30(44.1)$ & $58(51.3)$ & $<0.001$ \\
\hline Onset to admission (hours) & $24.0(7.0-48.0)$ & $30.0(14.3-83.0)$ & $32.0(9.0-57.0)$ & $36.0(16.3-72.0)$ & $<0.001$ \\
\hline Length of stay (days)* & $7.0(5.0-9.0)$ & $6.0(4.5-10.0)$ & $7.0(5.0-10.0)$ & $1.0(0.0-7.0)$ & $<0.001$ \\
\hline Admission to intervention (hours) ${ }^{*}$ & $120.0(6.5-163.4)$ & $149.6(120.0-192.0)$ & $144.0(24.0-168.0)$ & $8.0(4.0-149.6)$ & $<0.001$ \\
\hline Primary $\mathrm{PCl}$ & $10(4.7)$ & NA & NA & NA & NA \\
\hline Thrombolysis/fibrinolysis & $13(6.2)$ & NA & NA & NA & NA \\
\hline CAG performed & $112(53.1)$ & $27(31.8)$ & $44(64.7)$ & $90(79.6)$ & $<0.001$ \\
\hline Elective PCI/CABG & $30(14.2)$ & $10(11.8)$ & $11(16.2)$ & $35(31.0)$ & 0.001 \\
\hline
\end{tabular}

Values are $\mathrm{n}(\%)$ or mean $\pm \mathrm{SD}$, unless otherwise stated. Comparison of clinical profiles was performed using one-way analysis of variance for continuous variables and Pearson's $\chi^{2}$ test for categorical variables.

*Values are medians (Q1-Q3). Comparison was done using Kruskal-Wallis test.

†Comparison was performed using exact $\chi^{2}$ test.

‡Defined as angiographic two-vessel or three-vessel disease (stenoses $\geq 50 \%$ in at least two of the three major epicardial coronary arteries), which the cardiologist considered for stenting. ${ }^{27}$

BP, blood pressure; CABG, coronary artery bypass grafting; CAG, coronary angiography; eGFR, estimated glomerular filtration rate; HDLchol, high-density lipoprotein-cholesterol; HF, heart failure; LDL-chol, low-density lipoprotein-cholesterol; LVEF, left ventricular ejection fraction; LVH, left ventricle hypertrophy; NA, not available; NSTEMI, non-ST-elevation myocardial infarction; PCI, percutaneous coronary intervention; SCAD, stable coronary artery disease; STEMI, ST-elevation myocardial infarction; UA, unstable angina.

admission. Poor adherence to medications plays a significant role in mortality irrespective of underlying CAD diagnosis, particularly after hospital discharge. Although not formally tested, the same probably holds for lack of lifestyle adjustments.

The rates of mid-term mortality of acute and non-acute $\mathrm{CAD}$ in a low-income to middle-income Indonesian population were substantially higher compared with high-income or Western populations: for example 30.8\% of patients with STEMI from our study vs $7.1 \%$ from a 3-year follow-up study of patients with STEMI in UK. ${ }^{6}$ Another study from Australia with a 4-year mean follow-up observed an overall $10 \%$ all-cause mortality from patients with ACS and SCAD treated with PCI. ${ }^{14}$

Studies from the Western world usually focus on the more traditional risk factors such as age, ${ }^{6}$ diabetes mellitus, ${ }^{5}{ }^{6}$ systolic blood pressure, ${ }^{8}$ as well as adverse clinical profiles such as higher Killip class. ${ }^{6}$ It is established that the presence of hyperglycaemia on admission in non-diabetic patients was associated with an increased risk of 1-year mortality, similar to those with diabetes, even after correction for the use of guidelines-recommended treatment. ${ }^{7}$ Higher Killip class is an independent predictor of in-hospital mortality in patients with STEMI and non-ST elevation acute coronary syndrome. ${ }^{15} \mathrm{~A}$ meta-analysis ${ }^{16}$ and a 16-year follow-up study in the USA ${ }^{17}$ suggest that renal insufficiency (eGFR $<60 \mathrm{~mL} / \mathrm{min}$ ) is a significant predictor of all-cause mortality and cardiovascular mortality in general populations, including increased mortality after $\mathrm{ACS}^{18}$ or PCI. ${ }^{19}$

This explains the increased immediate mortality during hospitalisation observed in this cohort, independent 
Table 3 Adherence to medications and lifestyle adjustments after hospital discharge in all survivors compared by diagnosis

\begin{tabular}{|c|c|c|c|c|c|c|}
\hline & STEMI & NSTEMI & UA & SCAD & Total & \\
\hline At 30-day follow-up & $(n=178)$ & $(n=62)$ & $(n=62)$ & $(n=104)$ & $(n=406)$ & $P$ values \\
\hline \multirow{2}{*}{ Adherence to medications } & 76 (42.7) & $18(29.0)$ & $31(50.0)$ & $54(51.9)$ & 179 (44.1) & 0.025 \\
\hline & STEMI & NSTEMI & UA & SCAD & Total & \\
\hline At 30-day follow-up* & $(n=63)$ & $(n=14)$ & $(n=12)$ & $(n=19)$ & $(n=108)$ & $P$ values \\
\hline \multirow[t]{2}{*}{ Smoking cessation } & $40(63.5)$ & $5(35.7)$ & $7(58.3)$ & $12(63.2)$ & $64(59.3)$ & 0.291 \\
\hline & STEMI & NSTEMI & UA & SCAD & Total & \\
\hline At 3-year/ the end of follow-up & $(n=145)$ & $(n=41)$ & $(n=49)$ & $(n=81)$ & $(n=316)$ & $P$ values \\
\hline Reduced cholesterol diet† & $80(55.2)$ & $20(48.8)$ & $28(57.1)$ & $51(63.0)$ & $179(56.6)$ & 0.479 \\
\hline Reduced sodium/MSG diet & $76(52.4)$ & $20(48.8)$ & $28(57.1)$ & $51(63.0)$ & $175(55.4)$ & 0.364 \\
\hline
\end{tabular}

Values are $\mathrm{n}(\%)$. Comparison was performed using Pearson's $\chi^{2}$ test.

${ }^{*}$ Comparison was performed using exact $\chi^{2}$ test. For active smokers at baseline.

†Defined as none to $<3$ times per week consumption of deep-fried/fatty food and/or high-cholesterol seafood.

‡Defined as none to less than a quarter teaspoon $(<1500 \mathrm{mg})$ per day consumption of salt/MSG in daily food ${ }^{28}$ and/or none to $<3$ times per month consumption of local salty/MSG food.

MSG, monosodium glutamate; NSTEMI, non-ST-elevation myocardial infarction; SCAD, stable coronary artery disease; STEMI, ST-elevation myocardial infarction; UA, unstable angina.

from the lack of revascularisation. Unsurprisingly, in our study, patients with NSTEMI with poorer clinical profiles, greater prevalence of comorbidities, longer time intervals from admission to intervention, poorer adherence to after-discharge medications, and were less likely to undergo diagnostic CAG and elective PCI/CABG had the worst outcomes and considerably higher mortality

Table 4 Adherence to medications and total mortality within the disease subgroups and by sections of follow-up duration

\begin{tabular}{lrlr}
\hline & HR & 95\% Cl & P values \\
\hline \multicolumn{1}{l}{ Analyses within patient subgroups* } & & \\
\hline NSTEMI & 5.77 & 1.61 to 20.63 & 0.007 \\
\hline STEMI & 11.37 & 3.46 to 37.36 & $<0.001$ \\
\hline UA & 49.6 & 5.59 to 440.39 & $<0.001$ \\
\hline SCAD & 12.31 & 3.37 to 44.97 & $<0.001$ \\
\hline $\begin{array}{l}\text { Analyses in all patients } \dagger \\
\quad\end{array}$ & 10.28 & 5.51 to 19.18 & $<0.001$ \\
\hline $\begin{array}{l}\text { Total follow-up } \\
\text { Follow-up only } \\
\text { postdischarge }\end{array}$ & 9.74 & 5.04 to 18.85 & $<0.001$ \\
\hline
\end{tabular}

*Models were adjusted for age, sex, hypertension, hyperglycaemia, Killip class, eGFR, PCI/CABG, thrombolysis/anticoagulants, onset to admission, hospital stay, and rehospitalisation for MI, stroke or heart failure.

†Models were adjusted for age, sex, hypertension, hyperglycaemia, Killip class, cardiac biomarkers, ST-segment elevation, eGFR, PCI/CABG, thrombolysis/anticoagulants, onset to admission, hospital stay, rehospitalisation for MI, stroke or heart failure, socioeconomic status, and residence distance from the hospital.

CABG, coronary artery bypass grafting; eGFR, estimated glomerular filtration rate; MI, myocardial infarction; NSTEMI, nonST-elevation myocardial infarction; PCl, percutaneous coronary intervention; SCAD, stable coronary artery disease; STEMI, STelevation myocardial infarction; UA, unstable angina. rate compared with patients with STEMI, UA and SCAD (figure 4). A previous study also confirmed that cardiac mortality and outcomes were independently worse in patients with NSTEMI treated with PCI compared with those with stable angina, while the long-term outcomes of patients following PCI for STEMI were favourable, with similar outcomes as those with stable angina. ${ }^{20}$ Patients presenting with NSTEMI often have a higher prevalence of cardiovascular and non-cardiovascular comorbidities compared with patients with STEMI, ${ }^{20-22}$ and therefore the presence of such unmeasured confounders has been suggested to contribute to the adverse outcomes of the NSTEMI group.

We found that disease-related and patient-related predictors of all-cause mortality of patients with $\mathrm{CAD}$ in Indonesia were comparable with the predictors in high-income or Western populations. This study emphasises the difference in disease management due to lack of access to guidelines-recommended therapies and poor compliance to medication and lifestyle corrections. Particularly in Indonesia, financial and administrative barriers, transport difficulties, poor education and low awareness of the symptoms do play a role in delaying patients to get immediate reperfusion, as well as after-discharge medications for secondary prevention.

Due to local circumstances, transfer and admission of patients from remote areas to cardiac centres or hospitals with cardiac facilities in the city of Makassar is difficult. These situations most likely also exist in other traffic-clogged metropolitan areas in Indonesia. Apart from delayed access making a primary intervention futile, there was a considerably longer time lapse than the timeline for early reperfusion of $<120 \mathrm{~min}$ recommended by guidelines $^{23}$ : an average of 24 hours for STEMI and 30 hours for NSTEMI (see table 2). Nevertheless, we found that the prolonged delays from first onset to hospital admission 


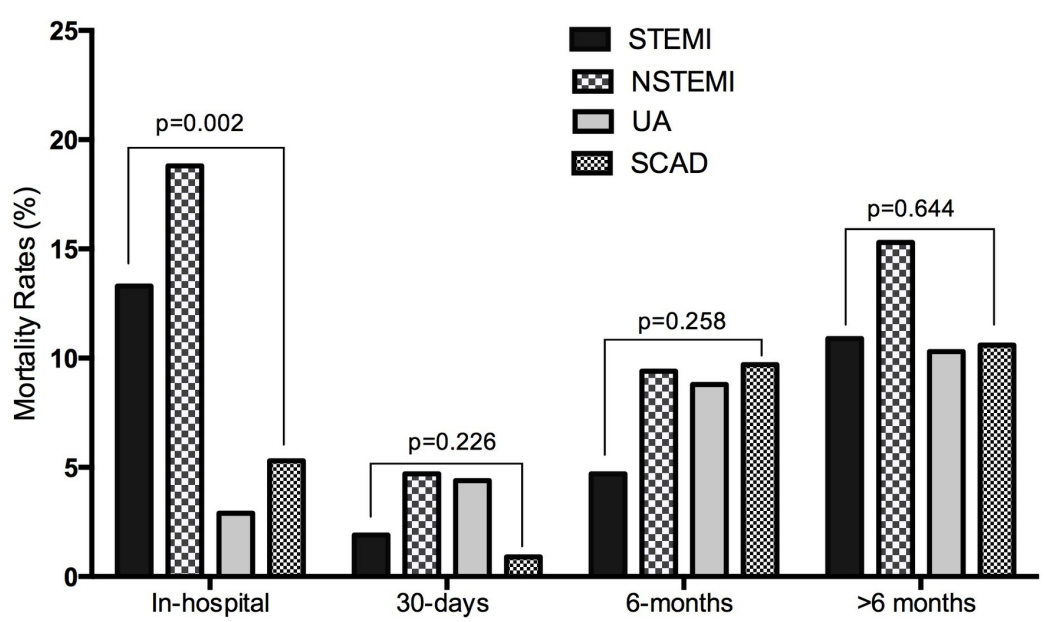

\begin{tabular}{|c|c|c|c|c|c|c|}
\hline Mortality & $\begin{array}{l}\text { STEMI } \\
(n=211)\end{array}$ & $\begin{array}{l}\text { NSTEMI } \\
(n=85)\end{array}$ & $\begin{array}{l}\text { UA } \\
(n=68)\end{array}$ & $\begin{array}{l}\text { SCAD } \\
(n=113)\end{array}$ & $\begin{array}{l}\text { Total } \\
(n=477)\end{array}$ & p-value \\
\hline In hospital & $28(13.3)$ & $16(18.8)$ & $2(2.9)$ & $6(5.3)$ & $52(10.9)$ & 0.002 \\
\hline Discharge -30 days & $4(1.9)$ & $4(4.7)$ & $3(4.4)$ & $1(0.9)$ & $12(2.5)$ & 0.226 \\
\hline$>30$ days -6 months & $10(4.7)$ & $8(9.4)$ & $6(8.8)$ & $11(9.7)$ & $35(7.3)$ & 0.258 \\
\hline$>6$ months -3 years ${ }^{*}$ & $23(10.9)$ & $13(15.3)$ & $7(10.3)$ & $12(10.6)$ & $55(11.5)$ & 0.644 \\
\hline Total & $65(30.8)$ & $41(48.2)$ & $18(26.5)$ & $30(26.5)$ & $154(32.3)$ & 0.007 \\
\hline
\end{tabular}

Figure 3 Rates of all-cause mortality among patients with coronary artery disease during the study period. NSTEMI, non-STelevation myocardial infarction; SCAD, stable coronary artery disease; STEMI, ST-elevation myocardial infarction; UA, unstable angina.

were insignificantly associated with higher medium-term, all-cause mortality in Indonesia (see figure 5). The plausible reason is that longer time delay from onset to admission and intervention was mostly associated with immediate in-hospital mortality, primarily in patients with acute myocardial infarction, that is, STEMI and NSTEMI

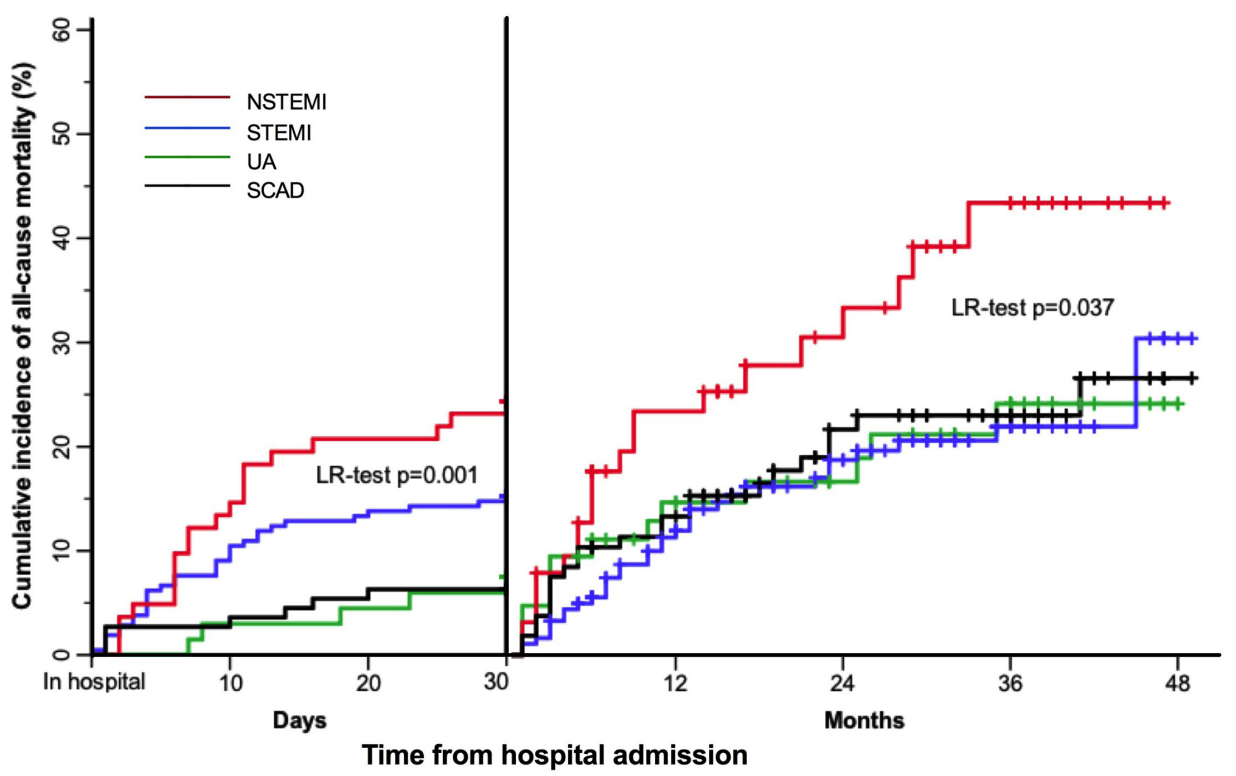

Figure 4 Kaplan-Meier curve presenting the cumulative incidence of all-cause mortality of patients with coronary artery disease over the follow-up period. Comparison was performed using log-rank (Mantel-Cox) test. LR test, log-rank test; NSTEMI, non-ST-elevation myocardial infarction; SCAD, stable coronary artery disease; STEMI, ST-elevation myocardial infarction; UA, unstable angina. 


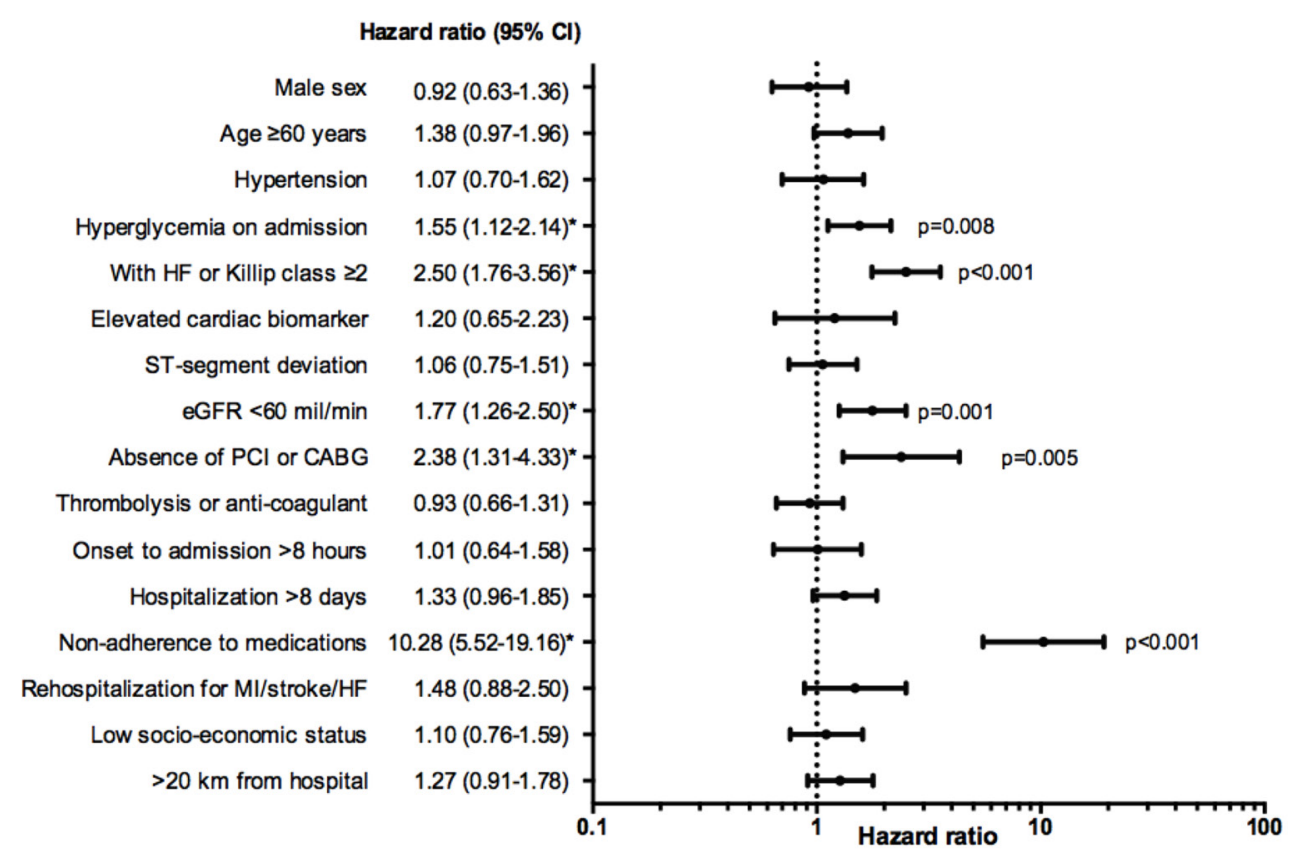

Figure 5 Forest plot presenting multivariable HRs for all-cause mortality during the study period. ${ }^{*} \mathrm{P}<0.05$. CABG, coronary artery bypass grafting; eGFR, estimated glomerular filtration rate; HF, heart failure; MI, myocardial infarction; PCI, percutaneous coronary intervention.

(figure 3). Accordingly, the rate of in-hospital mortality of patients with ACS in our study (12.6\%) was also higher than that of the Global Registry of Acute Coronary Events study from 94 hospitals in 14 countries in Europe, North and South America, Australia, and New Zealand (4.9\%). ${ }^{8}$

Patients undergoing PCI for ACS are known to have poorer short-term outcomes compared with stable CAD undergoing elective PCI. ${ }^{14}$ This is in line with our finding that $12.6 \%$ of patients with ACS and $5.3 \%$ of patients with $\mathrm{SCAD}$ died immediately after admission $(\mathrm{p}=0.029)$. A study from a high-income country also showed higher rates of in-hospital and medium-term, all-cause mortality in patients with ACS compared with SCAD (3\% vs $0 \%$ and $13 \%$ vs $7 \%$, respectively). ${ }^{14}$ However, a recent systematic review and meta-analysis of randomised clinical trials has highlighted that when compared with optimal medical therapy, PCI treatment did not reduce the risk of all-cause mortality, cardiovascular death, non-fatal myocardial infarction or repeat revascularisation in patients with stable $\mathrm{CAD}{ }^{24}$

At our cardiac centre, patients with ACS rarely underwent early invasive (ie, diagnostic angiography with intent to perform revascularisation) and ischaemia-guided strategies, while the majority of patients with stable CAD underwent elective PCI. Although an investment in infrastructure for early invasive and ischaemia-guided strategies seems expensive and cumbersome, improved adherence to guidelines for early intervention may result in a quick win of improved in-hospital and 30-day survival.

In this study, we found that adherence to medications of all survivors was lower compared with a large systematic review in resource-limited settings $(44.1 \%$ vs $57.5 \%){ }^{25}$
The factors that were considered to be attributable to poor adherence to cardiovascular medications in these resources-limited settings were lack of access, poor knowledge and negative perception of medications, side effects, high costs, change in symptoms, chronicity, comorbidities, complex treatment regimens, age, and family. ${ }^{25}$ We considered that all these factors were also applicable to our population in Indonesia. Additionally, in our study, geographical and regional distances were most likely responsible for the lack of access to after-care rehabilitation and secondary prevention. Hence, improved patient knowledge, through population-based seminar or using popular media, and improved performance of primary healthcare services in rural area may have an immediate effect on compliance and survival.

The present study has several limitations. All patients were required to sign a written informed consent form, which entailed excluding all patients with CAD who immediately died at the emergency department and ICCU. A face-to-face interview was not possible in all patients, as the majority $(51.2 \%)$ of patients live in rural, often very remote areas. In $239(50.1 \%)$ patients, interviews were carried out by telephone, which may have led to less accurate data than could have been obtained by a direct questionnaire interview. However, we always verified the answers at the next visit or by an extra telephone call using a different interviewer to reduce inaccuracies. In this study, we also used self-reported data from the survivors or family members to measure the clinical outcomes and after-discharge managements that may be introducing reporting bias. To minimise this bias, we verified the answers with the hospitals' 
medical records or family members' report. Finally, we excluded all suspected patients with normal CAG findings, which were mostly women. Thus, our study may have overlooked the minor group of myocardial infarction with no obstructive coronary atherosclerosis.

To our knowledge, this is the first study to identify predictors of medium-term mortality among patients with CAD in a resources-limited Indonesian population, and thus provides a general picture of risk stratification for clinical practitioners to recognise patients with $\mathrm{CAD}$ with poor prognosis. Rapid diagnosis and early risk stratification of patients with acute myocardial infarction (STEMI and NSTEMI) are mostly important in identifying patients in whom early interventions can result in improved clinical outcomes and reduced mortality risk. The increased burden of cardiovascular risk factors ${ }^{26}$ and the high mortality rate after hospital discharge in Indonesia imply that both primary and secondary prevention of CVD are still underperforming in this LMIC. Therefore, improvement in primary and secondary prevention of CVD in Indonesia is urgently required. Lastly, this study shows that generally in Indonesian patients with CVD, proper risk stratification and targeted-treatment policies can help clinicians improve patient outcomes. Improving patient access to proper facilities, optimising medication adherence and lifestyle adjustment will likely help reduce excess $\mathrm{CAD}$ mortality.

In conclusion, patients with $\mathrm{CAD}$ in Makassar, Indonesia of which represent a poor South-East Asian setting, have unacceptably high short-term and medium-term mortality rates. Both health policymakers and clinicians should help improve survival of Indonesian patients with CVD by organising compliance to guidelines-recommended therapy and improving adherence to medication and lifestyle corrections.

\section{Author affiliations \\ ${ }^{1}$ Department of Cardio-thoracic Surgery, AMC Heart Center, Academic Medical Center, University of Amsterdam, Amsterdam, The Netherlands \\ ${ }^{2}$ Department of Physiology, Faculty of Medicine, University of Hasanuddin, Makassar, Indonesia \\ ${ }^{3}$ Julius Center for Health Sciences and Primary Care, Universitair Medisch Centrum Utrecht, Utrecht, The Netherlands \\ ${ }^{4}$ Department of Cardiology, AMC Heart Center, Academic Medical Center, University of Amsterdam, Amsterdam, The Netherlands \\ ${ }^{5}$ Department of Cardiology and Vascular Medicine, Faculty of Medicine, University of Hasanuddin, Makassar, Indonesia}

Acknowledgements The authors gratefully acknowledge all participants, family members and advisers for all the support and contribution to this study. The staff of the Cardiovascular Care Unit (CVCU), RS Wahidin Sudirohusodo, Makassar, is acknowledged for their contributions to the success of this research project, and all research assistants for the data collection and data management. Thanks to Dian Sidik Arsyad, SKM, MKM, for providing figure 1 of this manuscript. Special thanks to Mrs Fithriany Harry S Farm, Apt, all research nurses, and nursing students in Puskesmas Batua (Batua Primary Healthcare Center), Makassar, for their dedication to complete the continuation of the follow-up.

Contributors CSPMU, $A Q$ and BAJMdM conceived the idea of the study and were responsible for the design of the study. $A Q, I M, I I$ and $M A$ were responsible for the data collection. $A Q$ was responsible for undertaking the data analysis and produced the tables and graphs. CSPMU, BAJMdM and JPSH provided input into the data analysis and interpretation. $\mathrm{AQ}$ has made the first complete paper draft, and the draft was then circulated repeatedly to CSPMU, BAJMdM and JPSH for critical revisions. All authors approved the final version of the manuscript.

Funding This work was supported by the Directorate General of Higher Education/ Direktorat Jenderal Pendidikan Tinggi (DIKTI), Ministry of National Education Republic of Indonesia (grant number 600/E4.4/K/2011, 2011).

Competing interests None declared.

Patient consent Obtained.

Ethics approval The study protocol was approved by the Ethics Committee and Institutional Review Board of the Faculty of Medicine, University of Hasanuddin, Makassar, Indonesia (letter number 030/H4.8.4.5.31/PP 36-KOMETIK/2013).

Provenance and peer review Not commissioned; externally peer reviewed.

Data sharing statement Data set available from the corresponding author at a. qanitha@amc.uva.nl/myaqanitha@gmail.com.

Open access This is an open access article distributed in accordance with the Creative Commons Attribution Non Commercial (CC BY-NC 4.0) license, which permits others to distribute, remix, adapt, build upon this work non-commercially, and license their derivative works on different terms, provided the original work is properly cited, appropriate credit is given, any changes made indicated, and the use is non-commercial. See: http://creativecommons.org/licenses/by-nc/4.0/.

\section{REFERENCES}

1. Yusuf S, Rangarajan S, Teo K, et al. Cardiovascular risk and events in 17 low-, middle-, and high-income countries. N Engl J Med 2014;371:818-27.

2. Ohira T, Iso H. Cardiovascular disease epidemiology in Asia. Circ J 2013;77:1646-52.

3. Lam CSP. Heart failure in Southeast Asia: facts and numbers. ESC Heart Fail 2015;2:46-9.

4. World Health Organization. Regional Office for South-East Asia. Noncommunicable Diseases in the South-East Asia Region: 2011 Situation and Response. New delhi 2011.

5. Prugger C, Wellmann J, Heidrich J, et al. Cardiovascular risk factors and mortality in patients with coronary heart disease. Eur $J$ Epidemiol 2008;23:731-7.

6. Rathod KS, Jones DA, Gallagher S, et al. Atypical risk factor profile and excellent long-term outcomes of young patients treated with primary percutaneous coronary intervention for STelevation myocardial infarction. Eur Heart J Acute Cardiovasc Care 2016;5:23-32.

7. Schiele F, Descotes-Genon V, Seronde MF, et al. Predictive value of admission hyperglycaemia on mortality in patients with acute myocardial infarction. Diabet Med 2006;23:1370-6.

8. Fox KA, Dabbous OH, Goldberg RJ, et al. Prediction of risk of death and myocardial infarction in the six months after presentation with acute coronary syndrome: prospective multinational observational study (GRACE). BMJ 2006;333:1091.

9. Kumar A, Cannon CP. Acute coronary syndromes: diagnosis and management, part I. Mayo Clin Proc 2009;84:917-38.

10. Grech ED, Ramsdale DR. Acute coronary syndrome: unstable angina and non-ST segment elevation myocardial infarction. BMJ 2003;326:1259-61.

11. Elbez $Y$, Cheong AP, Fassa AA, et al. Clinical outcomes in patients with stable coronary artery disease with vs. without a history of myocardial revascularization. Eur Heart J Qual Care Clin Outcomes 2016;2:23-32.

12. Pizzi C, Xhyheri B, Costa GM, et al. Nonobstructive versus obstructive coronary artery disease in acute coronary syndrome: a meta-analysis. J Am Heart Assoc 2016;5:e004185.

13. Ranthe MF, Carstensen L, Oyen N, et al. Family history of premature death and risk of early onset cardiovascular disease. J Am Coll Cardiol 2012;60:814-21.

14. Alcock RF, Yong AS, Ng AC, Ac N, et al. Acute coronary syndrome and stable coronary artery disease: are they so different? Longterm outcomes in a contemporary $\mathrm{PCl}$ cohort. Int $\mathrm{J}$ Cardiol 2013;167:1343-6.

15. El-Menyar A, Zubaid M, AlMahmeed W, et al. Killip classification in patients with acute coronary syndrome: insight from a multicenter registry. Am J Emerg Med 2012;30:97-103.

16. Matsushita K, van der Velde M, Astor BC, et al. Association of estimated glomerular filtration rate and albuminuria with all-cause and cardiovascular mortality in general population cohorts: a collaborative meta-analysis. Lancet 2010;375:2073-81.

17. Muntner P, He J, Hamm L, et al. Renal insufficiency and subsequent death resulting from cardiovascular disease in the United States. $J$ Am Soc Nephrol 2002;13:745-53. 
18. Gibson CM, Pinto DS, Murphy SA, et al. Association of creatinine and creatinine clearance on presentation in acute myocardial infarction with subsequent mortality. J Am Coll Cardiol 2003:42:1535-43.

19. Best PJ, Lennon R, Ting HH, et al. The impact of renal insufficiency on clinical outcomes in patients undergoing percutaneous coronary interventions. J Am Coll Cardiol 2002;39:1113-9.

20. Fath-Ordoubadi F, Spaepen E, El-Omar M, et al. Outcomes in patients with acute and stable coronary syndromes; insights from the prospective NOBORI-2 study. PLoS One 2014;9:e88577.

21. Montalescot G, Dallongeville J, Van Belle E, et al. STEMI and NSTEMI: are they so different? 1 year outcomes in acute myocardial infarction as defined by the ESC/ACC definition (the OPERA registry). Eur Heart J 2006;28:1409-17.

22. Balzi D, Di Bari M, Barchielli A, et al. Should we improve the management of NSTEMI? Results from the population-based "acute myocardial infarction in Florence 2" (AMI-Florence 2) registry. Intern Emerg Med 2013;8:725-33.

23 Kolh P, Windecker S, Alfonso F, et al. 2014 ESC/EACTS Guidelines on myocardial revascularization: the Task Force on Myocardial Revascularization of the European Society of Cardiology (ESC) and the European Association for Cardio-Thoracic Surgery (EACTS).
Developed with the special contribution of the European Association of Percutaneous Cardiovascular Interventions (EAPCl). Eur $J$ Cardiothorac Surg 2014;46:517-92.

24. Pursnani S, Korley F, Gopaul R, et al. Percutaneous coronary intervention versus optimal medical therapy in stable coronary artery disease: a systematic review and meta-analysis of randomized clinical trials. Circ Cardiovasc Interv 2012;5:476-90.

25. Bowry AD, Shrank WH, Lee JL, et al. A systematic review of adherence to cardiovascular medications in resource-limited settings. J Gen Intern Med 2011;26:1479-91.

26. Hussain MA, Al Mamun A, Peters SA, et al. The burden of cardiovascular disease attributable to major modifiable risk factors in Indonesia. J Epidemiol 2016;26:515-21.

27. Tonino PA, Fearon WF, De Bruyne B, et al. Angiographic versus functional severity of coronary artery stenoses in the FAME study fractional flow reserve versus angiography in multivessel evaluation. J Am Coll Cardiol 2010;55:2816-21.

28. American Heart Association. ANSWERS by heart, Lifestyle + Risk Reduction: High Blood Pressure. Why Should I Limit Sodium? 2017. http://www.heart.org/idc/groups/heart-public/@wcm/@hcm/documents/ downloadable/ucm_300625.pdf (accessed 22 Sep 2017). 